\title{
Comparison of Oral versus Intravenous Tranexamic Acid in Total Hip and Knee Arthroplasty
}

\author{
Jennifer Remington ${ }^{1}$, Rachana Patel ${ }^{1}$, William Stanfield ${ }^{1}$, Karen Kier $^{2}$ and Amber Baehr ${ }^{1}$ \\ 1. Department of Pharmacy, University Hospitals St. John Medical Center, 29000 Center Ridge Road, Westlake, Ohio 44145, USA \\ 2. College of Pharmacy, Ohio Northern University, 525 South Main Street, Ada, Ohio 45810, USA
}

\begin{abstract}
Objectives: The aim of this study is to compare blood loss from IV (intravenous) TXA (tranexamic acid) to PO (oral) TXA in THA (total hip arthroplasty) and TKA (total knee arthroplasty) at UHSJMC (University Hospitals St. John Medical Center). The primary endpoint is to compare the hemoglobin change $(\mathrm{g} / \mathrm{dL})$ in patients using IV versus PO TXA after THA or TKA. The secondary endpoints are to compare the amount of blood loss $(\mathrm{mL})$, amount of transfusions (units), and length of stay (days). Methodology: This is a retrospective chart review of patients who receive IV or PO TXA during a THA or TKA. Inclusion criteria are patients receiving IV TXA between September 1, 2018 and February 28, 2019 or patients receiving PO TXA between September 1, 2019 and February 29, 2020 for a THA or TKA. Patients will be excluded if they are less than 18 years old; patients who have a history of cognitive/psychosocial impairment, dementia, or language barrier, have had a deep vein thrombosis or pulmonary embolism within the past twelve months; have a history of deep vein thrombosis or pulmonary embolism being treated with anticoagulation; known congenital thrombophilia; or cardiac stent or ischemic stroke within one year. Primary endpoint will be analyzed using a standard independent $T$-test comparing mean change in hemoglobin. Secondary endpoints will be analyzed using descriptive statistics. Results: A total of 357 patients were reviewed for inclusion in the study with a total of 289 patients enrolled, 257 in the IV group and 32 in the PO group. There were 107 THA and 150 TKA included in the IV TXA group. There were 18 THA and 14 TKA included in the PO TXA group. The primary outcome of change in hemoglobin was $-2.721 \pm 1.04 \mathrm{~g} / \mathrm{dL}$ for the IV TXA group and $-2.513 \pm 1.09 \mathrm{~g} / \mathrm{dL}$ for the PO TXA group $(p<0.001)$. The secondary outcome of blood loss was $189.35 \pm 180.2 \mathrm{~mL}$ for the IV TXA group and $89.69 \pm 70.7 \mathrm{~mL}$ for the PO TXA group $(p<0.001)$. The secondary endpoint of length of stay was $2.02 \pm$ 1.05 days for the IV TXA group and $1.84 \pm 1.05$ days for the PO TXA group $(p<0.001)$. Only one patient received a blood transfusion of 2 units during this study therefore statistical tests could not be performed. A post hoc analysis was preformed comparing THA to TKA. Hemoglobin change, length of stay, and transfusions were non-inferior to each other but amount of blood loss was significantly more for THA versus TKA $(p<0.001)$. Conclusion: With the ease of use of PO tablets, possible cost savings, and longer half-life, the use of PO TXA may be more beneficial for select patients. Future studies with larger sample sizes, randomization, and lack of surgeon variability are still needed.
\end{abstract}

Key words: PO TXA, IV TXA, total hip arthroplasty, total knee arthroplasty, orthopedics.

\section{Introduction}

THA (total hip arthroplasty) and TKA (total knee arthroplasty) cause a significant amount of blood loss perioperatively which can lead to anemia and the need for blood transfusions. Therefore guidelines recommend the use of TXA (tranexamic acid) to minimize blood loss in THA and TKA. Furthermore,

Corresponding author: Jennifer Remington, PharmD, PGY1 Pharmacy Resident (omit Practice), Clinical Pharmacist, research fields: route of administration of TXA for TKA/THA, orthopedic surgery, efficacy of oral TXA to reduce blood loss in THA/TKA.
TXA is the only noted agent per the American Association of Hip and Knee Surgeons, American Society of Regional Anesthesia and Pain Medicine, American Academy of Orthopedic Surgeons, Hip Society, and Knee Society that is recommended to reduce blood loss in THA and TKA, making it standard of practice [1]. TXA is an antifibrinolytic agent with many FDA and off-label indications to prevent blood loss. TXA is generally well tolerated but the routes of administrations have different pharmacokinetics. PO (oral) TXA has a 2-hour onset, while IV is almost immediate. PO TXA has roughly 
an 11-hour half-life, while IV has only about 2 h [2, 3]. The most efficacious route of administration to prevent blood loss during a THA or TKA is still uncertain but IV and topical routes of administration are widely used. IV and topical TXA were found to reduce the risk of transfusions compared to placebo by $60 \%$ and $71 \%$, respectively [4]. Therefore, it is standard practice to administer IV or topical TXA prior to a THA or TKA.

The use of PO TXA prior to THA or TKA is not as widely used but previous studies have shown no difference between IV, topical, and PO TXA. Kayupov et al. [5] conducted a double bind, randomized, control trial comparing $\mathrm{PO} 1.95 \mathrm{~g}$ to IV $1 \mathrm{~g}$ TXA preoperatively. The primary outcome of reduction in hemoglobin was non-inferior between the two groups ( $p=0.0008$ ) (determined with use of equivalence testing, with $p<0.05$ demonstrating equivalence between treatments). Luo et al. [6] conducted a double blind, placebo controlled trial comparing PO 2 g to IV $20 \mathrm{mg} / \mathrm{kg}$ and topical $2 \mathrm{~g}$ TXA preoperatively. Their primary outcome was reduction in hemoglobin which showed non-inferiority between each group ( $p=0.73$ ). A meta-analysis of 7 studies showed no difference in hemoglobin change, blood loss, or amount of transfusions given between IV and PO TXA groups [6].

UHSJMC (University Hospitals St. John Medical Center) is a 204-bed nonprofit teaching community hospital that provides care to patients in northeast Ohio. Previously, UHSJMC standard TXA protocol for THA and TKA consists of a total of $2 \mathrm{~g}$ of IV TXA. Since September 2019, PO TXA 1,950 mg 90 min prior to surgery followed by an additional 1,950 mg 6-8 h postoperatively and 1,950 mg morning of post-operative day 1 has been utilized. The aim of this study is to compare blood loss from IV TXA to PO TXA in THA and TKA at UHSJMC.

\section{Materials and Methods}

\subsection{Study Design}

This is a retrospective chart review of patients who receive IV or PO TXA during a THA or TKA.

Inclusion criteria are patients receiving IV TXA between September 1, 2018 and February 28, 2019 or patients receiving PO TXA between September 1, 2019 and February 29, 2020 for a THA or TKA. Patients will be excluded if they are less than 18 years old; patients who have a history of cognitive/psychosocial impairment, dementia, or language barrier; have had a deep vein thrombosis or pulmonary embolism within the past twelve months; have a history of deep vein thrombosis or pulmonary embolism being treated with anticoagulation; known congenital thrombophilia; or cardiac stent or ischemic stroke within one year.

The primary endpoint is to compare the hemoglobin change $(\mathrm{g} / \mathrm{dL})$ in patients receiving IV versus PO TXA during a THA or TKA. The secondary endpoints are to compare the amount of blood loss $(\mathrm{mL})$, amount of transfusions (units), and length of stay (days) between the IV and PO TXA groups.

This study was approved by the University Hospitals institutional review board by expedited review.

\subsection{Statistical Analysis}

Primary endpoint will be analyzed using a standard independent $T$-test comparing mean change in hemoglobin. Secondary endpoints will be analyzed using descriptive statistics. Post hoc tests will be analyzed using an ANOVA (analysis of variance) test. Standard error of mean was completed to account for differences in patients between groups. A $p$ value of $<$ 0.05 was considered statistically significant.

\section{Results and Discussion}

\subsection{Results}

A total of 357 patients were reviewed for inclusion in the study. A total of 289 patients were enrolled, 257 in the IV group and 32 in the PO group (Fig. 1). Full baseline characteristics are listed in Table 1. There were a total of 107 THA and 150 TKA included in the 
IV TXA group (Fig. 2). There were a total of 18 THA and 14 TKA included in the PO TXA group (Fig. 3).

The primary outcome of change in hemoglobin was $-2.721 \pm 1.04 \mathrm{~g} / \mathrm{dL}$ for the IV TXA group and $-2.513 \pm$ $1.09 \mathrm{~g} / \mathrm{dL}$ for the PO TXA group $(p<0.001)$. The secondary outcome of blood loss was $189.35 \pm 180.2 \mathrm{~mL}$ for the IV TXA group and $89.69 \pm 70.7 \mathrm{~mL}$ for the PO TXA group $(p<0.001)$. The secondary endpoint

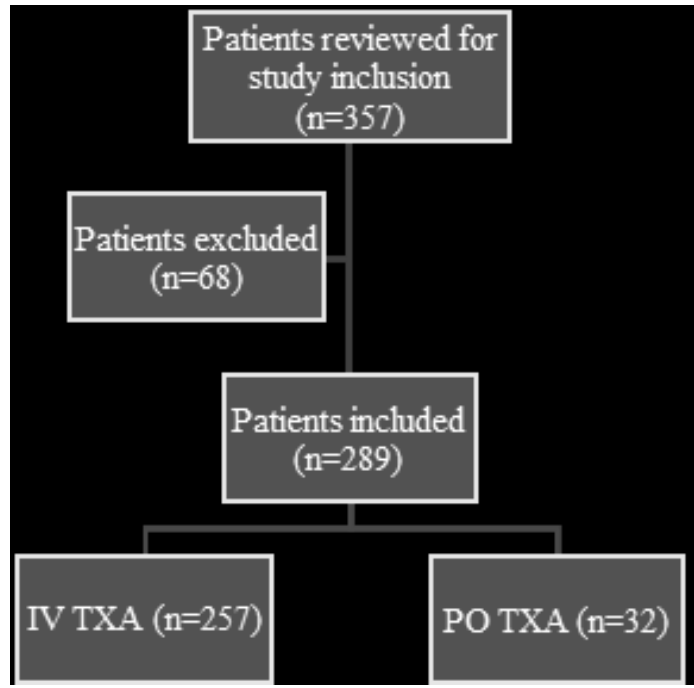

Fig. 1 Patient enrollment.

Table 1 Baseline demographics.

\begin{tabular}{lll}
\hline Baseline characteristic & PO $(n=32)$ & IV $(n=257)$ \\
\hline Age (years) & 64.6 & 70 \\
Male sex & $11(34.4 \%)$ & $98(38.1 \%)$ \\
THA & $18(56.3 \%)$ & $107(41.6 \%)$ \\
TKA & $14(43.7 \%)$ & $150(58.4 \%)$ \\
Pre-hemoglobin (g/dL) & $13.7 \pm 1.79$ & $13.6 \pm 1.42$ \\
Received PO iron & $2(6.3 \%)$ & $41(16.0 \%)$ \\
supplementation (patients) & & $28(10.9 \%)$ \\
$\begin{array}{l}\text { Received IV iron } \\
\text { supplementation (patients) }\end{array}$ & 0 & \\
\hline
\end{tabular}

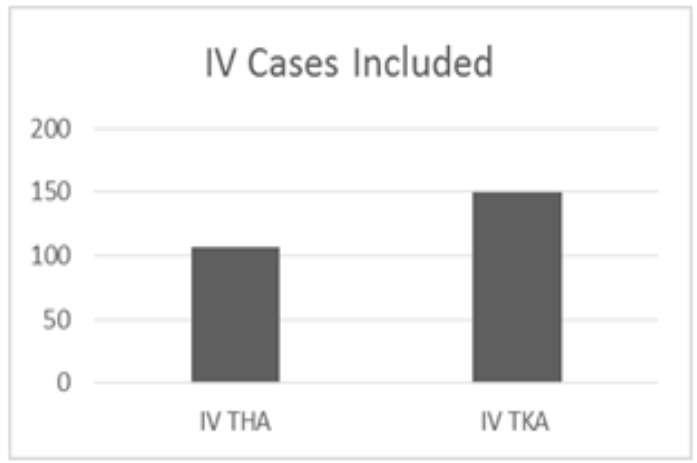

Fig. 2 IV cases included $(n=257)$.

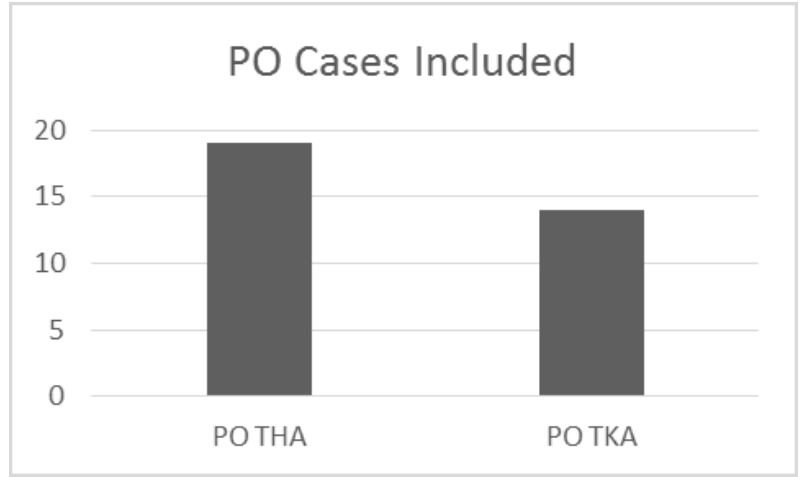

Fig. 3 PO Cases Included $(n=32)$.

of length of stay was $2.02 \pm 1.05$ days for the IV TXA group and $1.84 \pm 1.05$ days for the PO TXA group ( $p$ $<0.001)$. Only one patient received a blood transfusion of 2 units of pack red blood cells during this study therefore statistical tests could not be performed. A post hoc analysis was preformed comparing THA to TKA. Hemoglobin change, length of stay, and amount of transfusions were non-inferior to each other but amount of blood loss was significantly more for THA versus TKA $(p<0.001)$ (Table 3$)$.

\subsection{Discussion}

The use of TXA is standard of practice preoperatively for THA and TKA to prevent blood loss. The most efficacious route of administration has not been determined but topical and IV are widely used. PO TXA is not as widely used but recent studies have shown no difference between PO, IV, or topical routes [1, 4-6]. With the ease of use of PO tablets, possible cost savings, and longer half-life the use of PO TXA may be more beneficial for selected patients to prevent blood loss from a THA or TKA.

Previously, UHSJMC predominately used IV TXA for THA and TKA but since the summer of 2019 a few surgeons began using PO TXA. Due to recent literature showing non-inferiority and possible cost benefit, PO TXA was very appealing. Since the introduction to PO TXA, IV cost has been variable based on formulation utilized (Table 2). With the ease of use and possible benefit of using PO TXA, it may be more beneficial in select patients. 
Table 2 Cost of full course of therapy for PO and IV TXA.

\begin{tabular}{lll}
\hline & Cost & Cost (full course of therapy) \\
\hline PO $(650 \mathrm{mg}$ tablet $)$ & $\$ 2.66$ & $\sim \$ 26.00$ \\
IV $(1000 \mathrm{mg} / 10 \mathrm{~mL}$ vial $)$ & $\$ 4.55$ & $\sim \$ 9.00$ \\
IV $(1,000 \mathrm{mg} / 100 \mathrm{~mL}$ premix $)$ & $\$ 8.35$ & $\sim \$ 17.00$ \\
\hline
\end{tabular}

*McKesson 06/2020

Table 3 Primary and secondary outcomes.

\begin{tabular}{|c|c|c|c|c|}
\hline \multicolumn{5}{|l|}{ Primary outcome: } \\
\hline Change in hemoglobin (g/dL) & $\mathrm{N}$ & Mean & \multicolumn{2}{|l|}{$p$ value } \\
\hline IV & 257 & $-2.721 \pm 1.04$ & \multirow{2}{*}{$<0.001$} & \\
\hline PO & $30 *$ & $-2.513 \pm 1.09$ & & \\
\hline \multicolumn{5}{|l|}{ Secondary outcomes } \\
\hline Blood loss (mL) & $\mathrm{N}$ & Mean & $p$ value & Standard error of mean \\
\hline IV & 257 & $189.35 \pm 180.2$ & \multirow{2}{*}{$<0.001$} & 11.24 \\
\hline $\mathrm{PO}$ & 32 & $89.69 \pm 70.7$ & & 12.51 \\
\hline \multicolumn{5}{|l|}{ Length of stay (days) } \\
\hline IV & 257 & $2.02 \pm 1.05$ & \multirow{2}{*}{$<0.001$} & 0.07 \\
\hline PO & 32 & $1.84 \pm 1.05$ & & 0.19 \\
\hline
\end{tabular}

*2 patients excluded due to no pre-hemoglobin value provided.

In this study, 32 patients who received PO TXA prior to THA or TKA were compared to 257 patients who received IV TXA during the same time period one year prior. There were more patients in the IV group since only a few surgeons have begun to use PO TXA. The results showed the use of PO TXA to be statistically different from IV TXA in change in hemoglobin, blood loss, and length of stay. PO TXA has about an 11-hour half-life compared to IV which has only about $2 \mathrm{~h}$. Patients also receive three doses of PO TXA covering them for over $24 \mathrm{~h}$ compared to IV which is only given to the patient during surgery. This may have led to reduction in blood loss and hemoglobin. This all may have led to a quicker recovery and discharge.

Although currently there may not be a direct cost benefit of PO TXA, the ease of PO administration and decrease in length of stay could lead to a decrease in overall cost of hospital stay. This study also confirms past studies that PO TXA is equivalent to IV TXA and may even be superior to IV due to its pharmacokinetics.

This study has four limitations. First, this study was single centered limiting its external validity. Second, the PO TXA group had a small sample size compared to the IV group, but statistical tests controlled for this limitation by using standard error of the mean. Third, the IV group was all patients who received IV TXA for a THA and TKA regardless of the orthopedic surgeon. However, the PO group was only a few orthopedic surgeons. This may have led to differences in length of stay and amount of blood loss. Lastly, the amount of iron supplementation was reviewed but this was mostly provider specific and little information could be drawn from the results.

Studies have reviewed multiple different dosing strategies of oral TXA. A future study reviewing the most efficacious and safe dose of oral TXA is still needed. A future study could compare IV to PO TXA for the same orthopedic surgeons in both groups. In summary, PO TXA showed decrease in hemoglobin, less blood loss, and a shorter length of stay when compared to IV TXA at UHSJMC.

\section{Conclusions}

Current literature has shown non-inferiority between PO and IV TXA to prevent blood loss from 
THA and TKA [4-6]. A retrospective review of the use of IV and PO TXA at UHSJMC for THA and TKA showed a benefit to the use of PO TXA over IV. There was a significantly less decrease in hemoglobin, less blood loss, and a shorter length of stay when administering 1,950 mg $90 \mathrm{~min}$ prior to surgery followed by an additional 2 doses postoperatively. With the ease of use of PO tablets, possible cost savings, and longer half-life the use of PO TXA may be more beneficial for selected patients. Future studies with larger sample sizes, randomization, and limited surgeon variability are still needed.

\section{Acknowledgments}

The authors gratefully acknowledge University Hospitals St. John Medical Center orthopedic surgeons, nurses, and pharmacists for their continued support and enthusiasm throughout the research process.

\section{References}

[1] Fillingham, Y. A., Ramkumar, D. B., Jevsevar, D. S.,
Yates, A. J., Bini, S. A., Clarke, H. D., et al. 2018. "Tranexamic Acid Use in Total Joint Arthroplasty: The Clinical Practice Guidelines Endorsed by the American Association of Hip and Knee Surgeons, American Society of Regional Anesthesia and Pain Medicine, American Academy of Orthopaedic Surgeons, Hip Society, and Knee Society.” J Arthroplasty 33 (10): 3065.

[2] Micro Labs Limited. 2019. Tranexamic Acid Injection [package insert]. Basking Ridge.

[3] Amring Pharmaceuticals Inc. 2016. Tranexamic Acid Tablet [package insert]. Berwyn, PA.

[4] Chen, X., Zheng, F., Zheng, Z., Wu, X., and Wu, C. 2019. "PO vs. Intravenous Tranexamic Acid in Total-Knee Arthroplasty and Total Hip Arthroplasty: A Systematic Review and Meta-Analysis.” Medicine 98 (20): e15248.

[5] Kayupov, E., Fillingham, Y. A., Okroj, K., Plummer, D. R., Moric, M., Gerlinger, T. L., et al. 2017. "PO and Intravenous Tranexamic Acid Are Equivalent at Reducing Blood Loss Following Total Hip Arthroplasty: A Randomized Controlled Trial.” J Bone Joint Surg Am. 99 (5): 373-8.

[6] Luo, Z. Y., Wang, H. Y., Wang, D., Zhou, K., Pei, F. X., and Zhou, Z. K. 2018. "PO vs. Intravenous vs. Topical Tranexamic Acid in Primary Hip Arthroplasty: A Prospective, Randomized, Double-Blind, Controlled Study.” J Arthroplasty. 33 (3):786-93. 